(2) Open Access Full Text Article

\title{
Assessing the value of preoperative medical clearance in patients with primary rhegmatogenous retinal detachment
}

This article was published in the following Dove Press journal:

Clinical Ophthalmology

\author{
Rolake O Alabi' \\ Zachary A Turnbull ${ }^{2}$ \\ Peter G Coombs' \\ Yiyuan $\mathrm{Wu}^{3}$ \\ Anton Orlin' \\ RV Paul Chan ${ }^{4}$ \\ Szilard Kiss' \\ Donald J D'Amico' \\ Mrinali P Gupta' \\ 'Department of Ophthalmology, Weill \\ Cornell Medical College, New York, NY, \\ USA; ${ }^{2}$ Department of Anesthesiology, \\ Weill Cornell Medical College, New \\ York, NY, USA; ${ }^{3}$ Division of Biostatistics \\ and Epidemiology, Department of \\ Healthcare Policy \& Research, Weill \\ Cornell Medical College, New York, NY, \\ USA; ${ }^{4}$ Department of Ophthalmology \\ and Visual Sciences, Illinois Eye and Ear \\ Infirmary, University of Illinois at Chicago, \\ Chicago, IL, USA
}

Correspondence: Mrinali P Gupta Department of Ophthalmology, Weill Cornell Medical College, I 305 York Avenue, New York, NY 1002I, USA

$\mathrm{Tel}+$ I 6469622217

Fax + I 6469620600

Email mrp9003@med.cornell.edu
Purpose: To determine rates of intraoperative and postoperative systemic and ocular adverse events and establish the value of preoperative medical assessment in patients undergoing surgery for primary rhegmatogenous retinal detachment repair at a single academic center.

Patients and methods: Retrospective cohort study of 185 patients undergoing surgery for repair of primary rhegmatogenous retinal detachment (RRD) at a single academic center. Medical records were reviewed for medical comorbidities, completion of preoperative medical examination, anesthesia used during surgery, intraoperative adverse medical events, intraoperative ocular complications, and systemic and ocular postoperative complications. The main outcome of interest was the association of comorbidities and preoperative medical evaluation with intraoperative and postoperative complications.

Results: Approximately $48 \%$ of the patients presented with no medical comorbidities of interest. Formal preoperative evaluation by an independent medical provider was completed in $36 \%$ of the patients. Overall, intraoperative and postoperative systemic complications $(5.7 \%$ and $1 \%$, respectively) and intraoperative and postoperative ocular complications $(0.5 \%$ for both) were uncommon. Patients with a history of chronic heart failure (OR 24.5, $P=0.02$ ) or who received general anesthesia (OR 9.56, $P<0.001$ ) had increased risk of having experienced any intraoperative or postoperative complication. No relationship between preoperative medical evaluation and intraoperative and postoperative complications was observed.

Conclusion: Patients undergoing surgery for RRD repair presented with fewer medical comorbidities than previously reported in patients undergoing all vitreoretinal surgeries. Intraoperative and postoperative complications were uncommon and were increased in patients with chronic heart failure or who received general anesthesia. Complications were not significantly associated with preoperative evaluation by an independent medical provider.

Keywords: vitreoretinal surgery, retinal detachment, preoperative assessment, preoperative medical testing, adverse medical events, postoperative systemic adverse events

\section{Introduction}

Preoperative medical testing is routinely performed before intraocular surgery. Large retrospective and prospective studies and Cochrane database reviews of randomized clinical trials have demonstrated that routine preoperative testing in patients undergoing cataract surgery does not significantly reduce the risk of intraoperative or postoperative medical adverse events. ${ }^{1-3}$ Furthermore, the continued reliance on routine preoperative testing in cataract surgery patients has been 
shown to be associated with significant costs, reaching a potential estimated $\$ 45.4$ million annually for Medicare patients undergoing cataract surgery. ${ }^{4}$

The study of preoperative medical testing in vitreoretinal surgery has been much more limited. To date, a single retrospective study has evaluated the role of preoperative testing in postoperative systemic complications in all cases of vitreoretinal surgery performed at a single center. ${ }^{5}$ Shalwala et al showed postoperative adverse systemic events in $4 \%$ of the cases, with an increased risk among patients receiving general anesthesia and in patients with comorbidities including coronary artery disease, asthma, and chronic renal disease. Rates of intraoperative systemic complications were not reported. There was no sub-analysis for specific types of vitreoretinal surgeries-including urgent cases of primary rhegmatogenous retinal detachment (RRD) repair. ${ }^{2}$

To our knowledge, no previous studies have reported the rate of intraoperative systemic complications in vitreoretinal surgery or the value of preoperative testing in patients undergoing surgery for primary RRDs. We believe primary RRD repair patients represent a unique population warranting specific study for several reasons. (1) Patients who undergo surgery for primary RRD repair are likely younger and likely have fewer systemic comorbidities (including diabetes and hypertension) than patient populations undergoing cataract surgery and all types of vitreoretinal surgery that were the focus of prior studies. (2) In addition, primary RRD repair surgery, particularly for macula on retinal detachment, is generally performed urgently — often limiting the ability to obtain formal preoperative medical clearance. Yet, there are no studies evaluating the safety of this practice.

In this study, we completed a retrospective review of the medical charts and anesthesia records of all patients who underwent surgery for primary RRD at the Weill Cornell Medicine Department of Ophthalmology, and who had detailed intraoperative anesthesia and postoperative records available, to identify any adverse systemic health events in the intraoperative and 30-day postoperative period and to determine the relationship between these events and medical comorbidities and preoperative medical clearance.

\section{Materials and methods}

\section{Retrospective review}

This study was approved by the Weill Cornell Medical College Institutional Review Board and performed in concordance with the Health Insurance Portability and Accountability Act and the tenets put forth in the Declaration of Helsinki. The electronic medical record database of Weill Cornell Medical College was queried to identify all patients who underwent surgery for retinal detachment between February 2007 and April 2016 based on billing CPT codes $(67107,67018,67113)$. AO, RVPC, SK, DJD, and MPG performed the surgeries at Weill Cornell Medical College. Charts were reviewed to identify patients who underwent surgery for primary RRD repair. Specific inclusion criteria included: primary RRD repair, available preoperative and perioperative medical and anesthesia records, available intraoperative anesthesia records, and at least 30 days of postoperative follow-up. Exclusion criteria included complex retinal detachments (such as combination tractional-rhegmatogenous retinal detachments, macular hole retinal detachments, or keratoprosthesis-related retinal detachments), non-surgical repair of primary RRD (eg, pneumatic retinopexy), recurrent retinal detachment, and any prior intraocular surgery within 4 weeks of detachment repair. RRDs repaired by WCMC surgical faculty with private outpatient clinics were excluded due to lack of sufficient postoperative follow-up data.

The electronic medical record was retrospectively reviewed to collect the following data: age, sex, initial encounter setting, time from diagnosis to surgery, macula on/off status (defined as foveal detachment on examination or optical coherence tomography), presence or absence of comorbidities including hypertension, diabetes, cardiac disease (ie, coronary artery disease [CAD], arrhythmia, and chronic heart failure $[\mathrm{CHF}])$, stroke, respiratory disease (ie, asthma, chronic obstructive pulmonary disease [COPD]), chronic kidney failure, history of malignancy, history of anticoagulant use, smoking history (never, prior, or current smoking). Completion of formal preoperative medical examination (defined as history and physical examination completed by a medical provider other than the surgeon and completed prior to the day of surgery) was noted for all patients. Routine preoperative assessments completed on the day of surgery-most frequently by anesthesia providers - were not included in this study.

The surgical record was reviewed to determine type of surgery (scleral buckle and/or pars plana vitrectomy), anesthesia used (retrobulbar block, subtenon block, peribulbar block, monitored anesthesia care [MAC], and/or general anesthesia), and intraoperative ocular complications. The intraoperative anesthesia record and the electronic medical record were reviewed to identify systemic 
intraoperative or postoperative systemic complications including the following: bradycardia (heart rate $[\mathrm{HR}]<60$ ) or tachycardia $(\mathrm{HR}>100)$ requiring intervention, hypertension (blood pressure $[\mathrm{BP}]>140$ systolic or $\mathrm{BP}>90$ diastolic) requiring intervention, hypotension $(\mathrm{BP}<100$ systolic) with associated symptoms or requiring intervention, low oxygen $\left(\mathrm{O}_{2}\right)$ saturation (defined as $\mathrm{O}_{2}$ saturation $<88 \%$, a postoperative $\mathrm{O}_{2}$ requirement greater than patient's baseline, or $\mathrm{O}_{2}$ saturation requiring additional maneuvers), hypoglycemia (blood glucose $<60$ ), hyperglycemia requiring hospitalization, myocardial infarction (MI), CHF exacerbation requiring modification of medical management or hospitalization, arrhythmia, pulmonary complications (pulmonary embolism, pneumonia, and asthma exacerbation), respiratory failure requiring ventilation, inability to extubate (in general anesthesia cases), stroke, unanticipated postoperative hospitalization, and death. Intraoperative ocular complications which may relate to systemic status, such as hemorrhage or infection, were also included. Postoperative ocular complications such as recurrent retinal detachment (eg, proliferative vitreoretinopathy) and intraocular pressure elevation were not included as they do not correlate strongly with systemic health status which may be impacted by preoperative clearance.

\section{Statistical analysis}

Mean and standard deviation were calculated for case characteristics; median and mode were also determined, when relevant. Prism 6 (Graph Pad) software was used to perform the Chi-squared test to compare the rates of preoperative clearance in macula-on/off cases and to perform the Fisher's exact test to compare the rates of preoperative clearance in patients initially presenting in the emergency department or clinic. R software (version 3.4.3) was used to perform the Chi-squared test and Fisher's exact test to determine the association between individual risk factors (independent variables) and all complications (dependent variable). Risk factors analyzed included age, gender, smoking history, preoperative medical comorbidities (hypertension, CAD, CHF, arrhythmia, stroke, respiratory disease, chronic renal failure, history of malignancy), type of anesthesia used, and preoperative medical clearance. A combined complications outcome including all intraoperative and postoperative ocular complications, intraoperative adverse medical events, and postoperative systemic complications was used in analysis due to a low rate of complications and low sample size. Odds ratios (with 95\% confidence intervals) were calculated. All $P$-values were two-sided, and a $P$-value less than 0.05 was considered statistically significant.

\section{Results}

There were 193 cases (193 eyes in 185 patients) of surgical repair for primary RRD that met inclusion criteria and were analyzed for this study. The mean age of patients in the study was $52.2 \pm 17.7$, with approximately $39 \%$ of the patients aged 50 years of age or younger (Table 1). Patients in this cohort most commonly presented with a past medical history of hypertension $(31 \%)$, malignancy $(10 \%)$, coronary artery disease (9\%), and diabetes mellitus (9\%) (Table 2). Notably, $48 \%$ of the patients presented with no medical comorbidities of interest. The majority of detachments were first diagnosed in the outpatient setting (79\%), and the remainder were diagnosed in the emergency department (19\%) or inpatient settings (2\%). Approximately $60 \%$ of the cases were macula-

Table I Patient characteristics: demographics

\begin{tabular}{|l|l|}
\hline Sex & n (\%) \\
\hline $\begin{array}{l}\text { Male } \\
\text { Female }\end{array}$ & $\begin{array}{l}131(68) \\
62(32)\end{array}$ \\
\hline Age, years & $52.2 \pm 17.7$ \\
\hline Age distribution & $\mathbf{n ~ ( \% )}$ \\
\hline $\begin{array}{l}\leq 17 \text {-year-old } \\
\text { I8-50-year-old } \\
\geq 50 \text {-year-old }\end{array}$ & $\begin{array}{l}8(4) \\
68(35) \\
117(61)\end{array}$ \\
\hline
\end{tabular}

Table 2 Patient characteristics: medical comorbidities

\begin{tabular}{|l|l|}
\hline Comorbidity & n (\%) \\
\hline Hypertension & $60(3 \mathrm{I})$ \\
Coronary artery disease & $18(9)$ \\
Congestive heart failure & $3(2)$ \\
Arrhythmia & $6(3)$ \\
Diabetes mellitus & $17(9)$ \\
Lung disease & $15(8)$ \\
Transient ischemic attack & $2(1)$ \\
Stroke & $0(0)$ \\
Chronic renal failure & $4(2)$ \\
Cancer (all) & $20(10)$ \\
\hline Smoking history & \\
\hline Current smoker & $13(7)$ \\
Prior smoker & $43(22)$ \\
Never smoker & $132(68)$ \\
\hline
\end{tabular}

Note: a Smoking history unknown in 5 cases. 
on, while $40 \%$ were macula-off RRDs. The average time from diagnosis to surgery was $4 \pm 9$ days for macula-on detachments and $6 \pm 13$ days for macula-off detachments (Table S1).

Overall, a minority of patients (36\%) underwent formal preoperative medical evaluation (history and physical examination) with clearance from an independent medical provider (Table S2). Independent preoperative evaluation was completed in only $27 \%$ of the macula-on cases, which was significantly fewer than completed in macula-off cases (41\%, $P=0.04$, Table 3 ). There was no statistically significant difference between the rate of preoperative evaluation in cases initially diagnosed in the outpatient setting compared to in the emergency department $(P=0.18)$. The majority of surgeries were performed with MAC (79\%), while $21 \%$ of the surgeries were completed under general anesthesia (Table S2).

Intraoperative systemic (5.7\%, Table S3) and ocular complications $(0.5 \%$, Table $\mathrm{S} 4)$ were rare. Intraoperative systemic complications encountered include bradycardia or tachycardia requiring intervention $(2 \%)$, symptomatic hypotension or hypotension requiring intervention $(2 \%)$, or hyperglycemia requiring treatment $(0.5 \%$, Table $\mathrm{S} 3)$. The intraoperative ophthalmic complication encountered was choroidal hemorrhage. Overall rates of postoperative systemic complications (1\%) and ocular complications (1 patient [0.5\%] with vitreous hemorrhage) were similarly low. Both cases of postoperative systemic complications and the single case with a postoperative ocular complication had received preoperative medical clearance.

A history of chronic heart failure (OR 24.5, $P=0.02$ ) was associated with an increased risk of intraoperative or

Table 3 Preoperative clearance

\begin{tabular}{|c|c|}
\hline Overall & n (\%) \\
\hline Cleared & $69(36)$ \\
\hline Not cleared & $124(64)$ \\
\hline By age group & n (\%) \\
\hline Cleared, age $\leq 17$-year-old & $8(100)$ \\
\hline Cleared, age, I8-50-year-old & $24(35)$ \\
\hline Cleared, age $\geq 50$-year-old & $37(32)$ \\
\hline By macula status ${ }^{a}$ & n (\%) \\
\hline Cleared, macula-on & $21(27)$ \\
\hline Cleared, macula-off & $48(4 I)$ \\
\hline
\end{tabular}

Note: ${ }^{a} P<0.05$ using chi-squared test (Prism 6) comparing macula-on and maculaoff cases with or without preoperative clearance. postoperative ocular or systemic complications (Table 4) while CAD was associated with no increase in complication risk. While a history of arrhythmia (OR 6.77, $P=0.07$ ) or of any cardiac disease (OR 3.48, $P=0.06$ ) was associated with a trend toward increased risk of complications, all other preoperative comorbidities (hypertension, lung disease, and diabetes) were not associated with any increase or decrease in the risk of complications (Table 4). Similarly, other patient characteristics, such as age, sex, and smoking history, were not associated with increased risk of intraoperative or postoperative complications. Use of general anesthesia was associated with 9.56-fold increased odds of complications $(P<0.001)$. There was no significant association between independent preoperative medical clearance and risk of intraoperative or postoperative complications $(P=0.52$, Table 4$)$.

\section{Discussion}

The frequent need for emergent management of primary RRD cases, especially cases not yet involving the macula,

Table 4 Relationship between preoperative comorbidities and characteristics and any intraoperative and postoperative adverse events

\begin{tabular}{|c|c|c|c|}
\hline Preoperative comorbidity & OR & $95 \% \mathrm{Cl}$ of OR & $P$-value \\
\hline Hypertension & 1.54 & $0.48-4.56$ & 0.56 \\
\hline Cardiac disease (all) ${ }^{\mathrm{a}}$ & 3.48 & $0.86-11.7$ & 0.06 \\
\hline Coronary artery disease & 1.64 & $0.22-6.81$ & 0.64 \\
\hline Chronic heart failure & 24.5 & $1.88-802$ & 0.02 \\
\hline Arrhythmia & 6.77 & $0.78-40.6$ & 0.07 \\
\hline Lung disease & 2.05 & $0.27-8.73$ & 0.33 \\
\hline Diabetes & 0.81 & $0.03-4.54$ & 1.00 \\
\hline Preoperative characteristic & OR & $95 \% \mathrm{Cl}$ of $\mathrm{OR}$ & $P$-value \\
\hline Age, years & 1.01 & $0.98-1.04$ & 0.63 \\
\hline Sex ${ }^{b}$ & 1.08 & $0.31-3.23$ & 1.00 \\
\hline \multicolumn{4}{|l|}{ Smoking history } \\
\hline Current smoker & 2.92 & $0.37-14.0$ & 0.26 \\
\hline Prior smoker & 1.61 & $0.40-5.54$ & 0.26 \\
\hline History of anticoagulant use ${ }^{c}$ & 6.77 & $0.78-40.6$ & 0.07 \\
\hline General anesthesia & 9.56 & $3.11-33.4$ & $<0.001$ \\
\hline Preoperative medical clearance & 1.64 & $0.54-4.85$ & 0.52 \\
\hline
\end{tabular}

Notes: Adverse events defined as all intraoperative and postoperative systemic and ocular complications. All patients with complications had no history of chronic renal failure or malignancy. No patients in cohort presented with history of stroke. all patients with history of at least one of following conditions: coronary artery disease, myocardial infarction, congestive heart failure, arrhythmia, or other structural or valvular heart disease. ${ }^{b}$ Denotes odds ratio of being female relative to being male. 'History of anticoagulant use refers to documented history of taking any anticoagulation agent (ie, warfarin or non-vitamin $\mathrm{K}$ antagonist oral anticoagulants, NOACs) prior to surgery. The retrospective review was limited in its ability to reveal if, and for how long, anticoagulation agents were held prior to operations. 
often makes it difficult to obtain preoperative medical clearance by an independent medical provider. In some cases, surgery may be delayed to enable medical clearance. However, there are no studies that evaluate the risk of intraoperative and postoperative systemic complications in surgery for primary RRD. In this study, we sought to establish the rates of intraoperative and postoperative systemic and ocular complications among patients undergoing surgery for primary RRD and to determine whether patient characteristics, surgical characteristics, or preoperative medical clearance impact the risk of these complications. Our key findings were that (1) The rates of intraoperative and postoperative ocular and systemic complications were low for primary RRD repair; (2) Preoperative medical clearance testing was not associated with a lower risk of complications among all patients undergoing primary RRD repair; and (3) There was an increased risk of complications among patients with a history of congestive heart failure and among patients who underwent general anesthesia, with a trend toward increased risk among patients with a history of cardiac arrhythmia.

A single prior retrospective study by Shalwala et $\mathrm{al}^{5}$ evaluated risk of postoperative systemic complications in all cases of vitreoretinal surgery at a single institution. The rate of intraoperative systemic complications was not reported. Moreover, the rate of complications in the primary RRD subgroup was also not reported. Indeed, the population in the Shalwala et al study likely differs significantly from the primary RRD population given that vitreoretinal surgery is often undertaken for age-related conditions (eg, epiretinal membrane) and ocular manifestations of systemic conditions such as diabetes (ie, diabetic vitreous hemorrhage or tractional retinal detachment), both of which may correlate with increased risk of perioperative systemic complications.

In this study, we found a low rate of intraoperative systemic $(5.7 \%)$ and ocular $(0.5 \%)$ complications related to primary RRD repair. To our knowledge, this is the first report describing risk of intraoperative systemic complications in any study. As expected, the rate of postoperative systemic complications in this study was lower than that reported by Shalwala et al for all vitreoretinal surgeries ( $1 \%$ vs $4 \%$, respectively), likely a reflection of the disparate populations evaluated in these studies. Compared to a $73 \%$ medical clearance rate in Shalwala et al's study focused on preoperative medical testing in all cases of vitreoretinal surgery, ${ }^{5}$ only $36 \%$ of the primary RRD surgery patients in this study obtained formal medical clearance. As predicted, the mean age of patients in this study focused on primary RRD cases was younger than that in the study including all vitreoretinal surgery ${ }^{5}$ (52.2 compared to 58.5 years old). Patients in this study also less commonly exhibited co-morbid conditions-including coronary artery disease ( $9 \%$ compared to $18 \%)$, diabetes ( $9 \%$ compared to $37 \%$ ), and chronic kidney disease (2\% compared to 9\%) - than in the Shalwala et al study. ${ }^{5}$ Thus, subjects in this study represent, on average, a younger and healthier population (with fewer preoperative risk factors for cardiovascular complications) than the overall vitreoretinal surgery population. In addition, only $21 \%$ of the cases were completed under general anesthesia (compared to $41 \%$ in the Shalwala et al study), ${ }^{5}$ likely resulting in a decreased risk of potential cardiovascular and thromboembolic complications related to prolonged sedation compared to the general vitreoretinal surgery population. ${ }^{6}$ Altogether, these findings suggest that routine preoperative medical clearance may be even less critical in primary RRD cases than previously described for all cases of vitreoretinal surgery.

Among patients undergoing primary RRD repair, we found no association between preoperative medical clearance and increased or decreased risk of intraoperative or postoperative ocular or systemic complications. This finding is consistent with multiple large studies showing no benefit of preoperative clearance testing among all patients undergoing cataract surgery ${ }^{3-5}$ and among all patients undergoing vitreoretinal surgery. ${ }^{2}$ Nonetheless, preoperative clearance with at least a history and physical by an independent physician is still routinely performed for both intraocular surgeries. Moreover, additional preoperative tests (ie, basic or comprehensive metabolic panel, complete blood count, coagulation studies, electrocardiography, chest X-ray) are frequently completed as part of routine preoperative medical evaluation and are associated with significant costs to the health care system. Thus, careful consideration of the medical necessity and costeffectiveness of preoperative medical evaluation and testing remains important. Indeed, it seems plausible that, like routine history and physical, routine preoperative medical testing may be less critical in this relatively young, healthy cohort undergoing primary RRD repair with few complications. Further studies are necessary to evaluate this further. In the present study, the complete preoperative medical clearance records (including preoperative medical testing) for a majority of patients who underwent clearance were not available for review. 
This study found that among all comorbidities considered, only a history of chronic heart failure was associated with increased risk of complications. This is in contrast to the Shalwala et al study in which CAD, asthma, and chronic renal failure were associated with increased postoperative systemic complications. ${ }^{5}$ In both the current study and the prior study, use of general anesthesia was associated with increased complications. Based on these findings, physicians may consider these risk factors and balance them with the risk to vision with delay in surgery when discussing the option of prompt surgery versus delayed surgery (to enable formal medical clearance) in patients undergoing primary RRD repair.

The standard limitations of retrospective, observational studies apply to this study. In addition, the small sample size and resulting low power (of note, the chi-squared test used had a power of 0.16 to detect a difference of $12 \%$ in complication rates between cases with or without preoperative clearance), as well as the tertiary care setting of this study may limit the generalizability of these findings. Nevertheless, the higher rate of primary RRD cases observed in male patients than in female patients in our study is consistent with the higher overall incidence of RRD seen in male patients in many previous large epidemiological studies. ${ }^{7-16}$ In addition, as in prior studies, ${ }^{7-14,16-19}$ the rate of RRD increased with increasing age in this study population. Finally, the described frequency of macula-off RRDs $(60 \%)$ in this study is consistent with the previously reported macula-off rates ranging from $40 \%$ to $60 \%$ in Western studies. ${ }^{7,8,17}$ Cumulatively, this suggests that, despite the limited sample size, the current study population appears representative of the larger populations of RRD previously studied. Finally, due to the low rate of complications and the small sample size of this study, subgroup analyses of interest (for example, the utility of preoperative medical clearance according to baseline patient characteristics or surgery characteristics) were not possible. It is possible that although preoperative medical clearance did not reduce risk of complications in all primary RRD patients, those with certain systemic comorbidities or those undergoing general anesthesia may benefit from such evaluations. Caution must be taken before applying these findings to individual patients, especially those with significant medical comorbidities. Our results suggest, at best, that preoperative clearance by an independent provider may not be uniformly necessary. Future larger studies are necessary to determine the definitive value of preoperative clearance in primary RRD patients with and without medical comorbidities.
In conclusion, in this study, the frequency of intraoperative and postoperative systemic complications was low among patients undergoing surgery for primary RRD, and chronic heart failure and use of general anesthesia were associated with increased risk of complications. In the population of all patients undergoing primary RRD repair, a lack of completion of preoperative medical clearance was not associated with any increase in the risk of intraoperative and postoperative complications or adverse events. In light of the lack of prior reports evaluating intraoperative systemic complications during vitreoretinal surgery or any complications in the setting of surgery for primary RRD, these findings and the rates of complications reported herein may inform the discussion between the surgeon and patient with regard to the need for preoperative medical clearance for primary RRD repair - especially in the setting of urgent macula-on RRD cases. This paper lends support to the common practice of proceeding with surgery in macula-on primary RRD cases without antecedent formal preoperative medical clearance. Further, larger studies are necessary to determine the effectiveness and cost-effectiveness of preoperative medical clearance in patients undergoing primary RRD repair, including in subpopulations within this group.

\section{Ethics approval and informed consent}

This study was conducted after approval from the Institutional Review Board at Weill Cornell Medical College (New York, NY, USA). Individual patient consent was not required since this was a retrospective study and since all patient data were de-identified.

\section{Acknowledgment}

The authors would like to acknowledge the National Institutes of Health grant T32GM007739 to the TriInstitutional MD-PhD program (ROA), the Clinical and Translational Science Center at Weill Cornell Medical College Grant 1-UL1-TR002384-01, and an unrestricted departmental grant from Research to Prevent Blindness (AO, DJD, SK, and MPG).

\section{Disclosure}

Dr Robison Vernon Paul Chan reports personal fees from Allergan, Alcon, Visunex Medical Systems, Beyeonics, and Genentech outside the submitted work. Dr Szilard Kiss reports personal fees and a stock grant from Adverum, and personal fees from Alcon, Optos, RegenXBio, 
BioMarin, Regeneron, Novartis, Genentech/Roche, and Fortress Bio during the conduct of the study. Dr Donald J. D'Amico reports grants from Research to Prevent Blindness, Inc. during the conduct of the study. The authors report no other conflicts of interest in this work.

\section{References}

1. Schein OD, Katz J, Bass EB, et al. The value of routine preoperative medical testing before cataract surgery. Study of Medical Testing for Cataract Surgery. N Engl J Med. 2000;342(3):168-175. doi:10.1056/ NEJM200001203420304

2. Keay L, Lindsley K, Tielsch J, Katz J, Schein O. Routine preoperative medical testing for cataract surgery. Cochrane Database Syst Rev. 2009;2:CD007293. doi:10.1002/14651858.CD007293.pub2

3. Keay L, Lindsley K, Tielsch J, Katz J, Schein O. Routine preoperative medical testing for cataract surgery. Cochrane Database Syst Rev. 2012;3:CD007293. doi:10.1002/14651858.CD007293.pub3

4. Chen CL, Clay TH, McLeod S, Chang H-YP, Gelb AW, Dudley RA. A revised estimate of costs associated with routine preoperative testing in medicare cataract patients with a procedure-specific indicator. JAMA Ophthalmol. 2018;136(3):231-238. doi:10.1001/ jamaophthalmol.2017.6372

5. Shalwala A, Hwang RY, Tabing A, Sternberg P, Kim SJ. The value of preoperative medical testing for vitreoretinal surgery. Retina (Philadelphia Pa). 2015;35(2):319-325. doi:10.1097/IAE.0000000000000306

6. McCannel CA, Nordlund JR, Bacon D, Robertson DM. Perioperative morbidity and mortality associated with vitreoretinal and ocular oncologic surgery performed under general anesthesia. Trans Am Ophthalmol Soc. 2003;101:209-213; discussion 213-215.

7. Rowe JA, Erie JC, Baratz KH, et al. Retinal detachment in Olmsted County, Minnesota, 1976 through 1995. Ophthalmology. 1999;106 (1):154-159. doi:10.1016/S0161-6420(99)90018-0

8. Van de Put MAJ, Hooymans JMM, Los LI; Dutch Rhegmatogenous Retinal Detachment Study Group. The incidence of rhegmatogenous retinal detachment in The Netherlands. Ophthalmology. 2013;120 (3):616-622. doi:10.1016/j.ophtha.2012.09.001
9. Park SJ, Choi N-K, Choi N-K, Park KH, Woo SJ. Five year nationwide incidence of rhegmatogenous retinal detachment requiring surgery in Korea. PLoS One. 2013;8(11):e80174. doi:10.1371/journal. pone. 0080174

10. Chen S-N, Lian I-B, Wei Y-J. Epidemiology and clinical characteristics of rhegmatogenous retinal detachment in Taiwan. Br J Ophthalmol. 2016;100(9):1216-1220. doi:10.1136/bjophthalmol-2015-307481

11. Mitry D, Charteris DG, Yorston D, et al. The epidemiology and socioeconomic associations of retinal detachment in Scotland: a two-year prospective population-based study. Invest Ophthalmol Vis Sci. 2010;51(10):4963-4968. doi:10.1167/iovs.10-5400

12. Polkinghorne PJ, Craig JP. Northern New Zealand Rhegmatogenous Retinal Detachment Study: epidemiology and risk factors. Clin Experiment Ophthalmol. 2004;32(2):159-163. doi:10.1111/j.14429071.2004.00003.x

13. Curti S, Coggon D, Baldasseroni A, et al. Incidence rates of surgically treated rhegmatogenous retinal detachment among manual workers, non-manual workers and housewives in Tuscany, Italy. Int Arch Occup Environ Health. 2014;87(5):539-545. doi:10.1007/ s00420-013-0894-5

14. Chou S-C, Yang C-H, Lee C-H, et al. Characteristics of primary rhegmatogenous retinal detachment in Taiwan. Eye (Lond). 2007;21 (8):1056-1061. doi:10.1038/sj.eye.6702397

15. Jalali S, Yorston D, Shah NJ, et al. Retinal detachment in south Indiapresentation and treatment outcomes. Graefes Arch Clin Exp Ophthalmol. 2005;243(8):748-753. doi:10.1007/s00417-004-1085-6

16. Mowatt L, Shun-Shin G, Price N. Ethnic differences in the demand incidence of retinal detachments in two districts in the West Midlands. Eye (Lond). 2003;17(1):63-70. doi:10.1038/sj.eye.6700245

17. Mitry D, Charteris DG, Fleck BW, Campbell H, Singh J. The epidemiology of rhegmatogenous retinal detachment: geographical variation and clinical associations. Br J Ophthalmol. 2010;94(6):678-684. doi:10.1136/bjo.2009.157727

18. Limeira-Soares PH, Lira RPC, Arieta CEL, Kara-José N. Demand incidence of retinal detachment in Brazil. Eye (Lond). 2007;21 (3):348-352. doi:10.1038/sj.eye.6702202

19. Li X. Beijing rhegmatogenous retinal detachment study group. Incidence and epidemiological characteristics of rhegmatogenous retinal detachment in Beijing, China. Ophthalmology. 2003;110 (12):2413-2417. doi:10.1016/s0161-6420(03)00867-4 


\section{Supplementary materials}

Table SI Preoperative assessment characteristics

\begin{tabular}{|l|l|}
\hline Macula status & $\mathbf{n}(\%)$ \\
\hline Macula-on & $77(40)$ \\
Macula-off & $\mathrm{I} 16(60)$ \\
\hline Average time from diagnosis to surgery, Mac on (days) & $4 \pm 9$ \\
Median & $\mathrm{I}$ \\
Mode & 0 \\
Average time from diagnosis to surgery, Mac off (days) & $6 \pm 13$ \\
Median & $\mathrm{I}$ \\
Mode & $\mathrm{I}$ \\
\hline Initial encounter setting & \\
Clinic & \\
Emergency room & $152(79)$ \\
In-patient & $36(19)$ \\
\hline
\end{tabular}

Note: ${ }^{a}$ Encounter setting unknown in 2 cases.

Table S2 Surgical characteristics

\begin{tabular}{|l|l|}
\hline Surgery type & n (\%) \\
\hline Pars plana vitrectomy (PPV) & $132(68)$ \\
Scleral buckle (SB) & $40(2 \mathrm{I})$ \\
SB+PPV & $2 \mathrm{I}(\mathrm{II})$ \\
\hline Anesthesia used & $\mathbf{n}(\%)$ \\
\hline General anesthesia & $40(2 \mathrm{I})$ \\
Monitored anesthesia care & $153(79)$ \\
\hline Type of block used & $\mathbf{n}(\%)$ \\
\hline None & $35(18)$ \\
Retrobulbar & $140(73)$ \\
Subtenon & $4(2)$ \\
Peribulbar & $14(7)$ \\
\hline
\end{tabular}

Clinical Ophthalmology

\section{Publish your work in this journal}

Clinical Ophthalmology is an international, peer-reviewed journal covering all subspecialties within ophthalmology. Key topics include: Optometry; Visual science; Pharmacology and drug therapy in eye diseases; Basic Sciences; Primary and Secondary eye care; Patient Safety and Quality of Care Improvements. This journal is indexed on PubMed

Submit your manuscript here: https://www.dovepress.com/clinical-ophthalmology-journal
Table S3 Intraoperative adverse events or complications

\begin{tabular}{|c|c|}
\hline Intraoperative complications & n (\%) \\
\hline Bradycardia or tachycardia $^{a}$ & $4(2)$ \\
\hline Hypotension ${ }^{\mathrm{b}}$ & $4(2)$ \\
\hline Hypertension $^{c}$ & $2(1)$ \\
\hline Arrhythmia & $0(0)$ \\
\hline Myocardial infarction & $0(0)$ \\
\hline Congestive heart failure flare & $0(0)$ \\
\hline Desaturation & $0(0)$ \\
\hline Respiratory complications & $0(0)$ \\
\hline Respiratory failure $^{d}$ & $0(0)$ \\
\hline Stroke/CVA & $0(0)$ \\
\hline Hypoglycemia & $0(0)$ \\
\hline Hyperglycemia & I (0.5) \\
\hline Inability to extubate (general anesthesia cases) & $0(0)$ \\
\hline Postoperative hospitalizations & $0(0)$ \\
\hline Death & $0(0)$ \\
\hline
\end{tabular}

Notes: a Bradycardia or tachycardia requiring intervention. ${ }^{b}$ Hypotension defined as $\mathrm{BP}<100$ systolic with symptoms or requiring intervention. ${ }^{c}$ Hypertension requiring intervention. ${ }^{\mathrm{d}}$ Respiratory failure requiring ventilation.

Table S4 Other associated complications

\begin{tabular}{|l|l|}
\hline Ocular complications & n (\%) \\
\hline Intraoperative $^{\mathrm{a}}$ & $\mathrm{I}(0.5)$ \\
Postoperative $^{\mathrm{b}}$ & $\mathrm{I}(0.5)$ \\
\hline Systemic complications $^{\mathrm{s}}$ & $\mathbf{n}(\%)$ \\
\hline Postoperative $^{\mathrm{c}}$ & $2(\mathrm{I})$ \\
\hline
\end{tabular}

Notes: ${ }^{a}$ Intraoperative ocular complication was choroidal hemorrhage. ${ }^{\mathrm{b}}$ Postoperative ocular complication was vitreous hemorrhage. 'Postoperative systemic complications (2) were fevers with myalgias (I) and deep vein thrombosis and pulmonary embolism (I).

\section{Dovepress}

Central and CAS, and is the official journal of The Society of Clinical Ophthalmology (SCO). The manuscript management system is completely online and includes a very quick and fair peer-review system, which is all easy to use. Visit http://www.dovepress.com/ testimonials.php to read real quotes from published authors. 